\title{
Rapid detection of the red fire ant Solenopsis invicta (Hymenoptera: Formicidae) by loop-mediated isothermal amplification
}

\author{
Nobuyoshi Nakajima ${ }^{1}$ [ $\cdot$ Yoshiko Sakamoto $^{1} \cdot$ Koichi Goka $^{1}$
}

Received: 24 December 2018 / Accepted: 7 May 2019 / Published online: 22 May 2019

(c) The Author(s) 2019

\begin{abstract}
We developed a loop-mediated isothermal amplification (LAMP) assay to detect the red imported fire ant, Solenopsis invicta Buren, an invasive species in Japan. We designed species-specific LAMP primers on the basis of the nucleotide sequence of the $S$. invicta mitochondrial cytochrome $c$ oxidase subunit 1 region. The system we developed has a sensitivity and specificity enabling detection of one $S$. invicta in a mixed sample including nine individuals of a non-target ant species. We were easily able to detect the $S$. invicta DNA visually based on the turbidity of the reaction mixture. This rapid and easy LAMP assay system provides a powerful tool for the early detection and identification of S. invicta in invaded regions.
\end{abstract}

Keywords Invasive alien species $\cdot$ Solenopsis invicta $\cdot$ Loop-mediated isothermal amplification $\cdot$ COXI

\section{Introduction}

The red imported fire ant, Solenopsis invicta Buren (Hymenoptera: Formicidae), has been documented as entering Japan within ship cargo since June 2017. S. invicta queens and workers have been captured around international port areas, as well as in inland areas, and have even been seen in premises (Ministry of the Environment 2017; http://www. env.go.jp/nature/dobutsu/fireant.html\#ABOUT). Although no established $S$. invicta nests have been found, it is believed to be only a matter of time before this species will naturalize in Japan.

Early detection is an important strategy to prevent $S$. invicta expansion. Because $S$. invicta may be artificially introduced across broad geographic regions via commercial transport, wider monitoring by officials and citizens is needed. However, non-experts cannot easily identify $S$. invicta based on morphological characteristics alone. In fact, we ourselves have needed specialists to confirm our identification of $S$. invicta, a process requiring at least several days. Therefore, it is crucial to develop a rapid and easy procedure to identify S. invicta.

Nobuyoshi Nakajima

naka-320@nies.go.jp

Center for Environmental Biology and Ecosystems, National Institute for Environmental Studies, Onogawa 16-2,

Tsukuba, Ibaraki 305-8506, Japan
Recently, Valles et al. (2016) developed a lateral-flow immunoassay detection system for $S$. invicta identification by raising monoclonal antibodies against $S$. invicta-specific venom proteins. However, that system requires protein from at least five workers to identify the species. Because the number of $S$. invicta individuals captured in the field during the early stages of invasion will be very small, the sensitivity of that immunoassay system is likely insufficient.

We considered that the loop-mediated isothermal amplification (LAMP) assay (Notomi et al. 2000) may enable easy and rapid detection of $S$. invicta. The assay is a single-tube isothermal technique for DNA amplification. Because the assay procedure is simple and rapid, LAMP has proven to be a powerful tool for monitoring invasive species. For example, Ide et al. (2018) developed a LAMP assay protocol to detect another invasive ant, Linepithema humile (Mayr).

In the present study, we designed S. invicta-specific LAMP primers and evaluated their specificity by performing LAMP assay amplification of DNA extracted from whole or partial individuals of $S$. invicta and several other ant species.

\section{Materials and methods}

\section{Ant samples}

Solenopsis invicta workers were collected from the Tokyo, Nagoya, and Kobe Ports in June and July 2017. They were 
stored individually at $-20{ }^{\circ} \mathrm{C}$ in a microtube until DNA extraction. Workers of Cardiocondyla sp., Crematogaster osakensis, Formica japonica, Monomorium chinense, Pheidole noda, Solenopsis japonica, Temnothorax sp., and Tetramorium tsushimae were collected from Tokyo and Tsukuba City, Japan in July 2017. These eight ant species are commonly found in port areas and are likely to be mistakenly identified as $S$. invicta. These samples were immersed in $99.5 \%$ ethanol and stored at $-20{ }^{\circ} \mathrm{C}$ until DNA extraction.

\section{Primer design}

In this study, we designed $S$. invicta-specific LAMP primers based on several genetically distinct populations collected at Japanese ports by the Ministry of the Environment. We selected the mitochondrial cytochrome $c$ oxidase subunit 1 (COXI) gene as a target sequence. In arthropods, the nucleotide sequence of the $C O X 1$ region is highly divergent, and therefore is useful for species identification (Valles and Porter 2003). The nucleotide sequence of mitochondrial COXI was obtained from the mitochondrial genome data of $S$. invicta registered in NCBI (https://www.ncbi.nlm.nih.gov/ nuccore/NC_014672.1; accession no. NC_014672). Based on the sequence data, we designed seven sets of LAMP primer candidates using Primer Explorer Ver. 5.0 (https ://primerexplorer.jp/v5_manual/index.html) under default conditions.

\section{Evaluation of species specificity using purified DNA}

DNA was isolated through SDS-phenol extraction with a modified RNA isolation method described by Nakajima et al. (1988). The whole body of each ant was separately homogenized with a mortar and pestle in $200 \mu \mathrm{L}$ of extraction buffer (50 mM Tris-HCl (pH 8.0), 10 mM EDTA, and $1 \%$ SDS) and an equivalent amount of water-saturated phenol. The extracts were incubated at $60{ }^{\circ} \mathrm{C}$ for $10 \mathrm{~min}$. After centrifugation at $20,000 \times g$, the upper phases were transferred to fresh microcentrifuge tubes and sequentially extracted in phenol:chloroform $(1: 1)$ and then chloroform. DNA was recovered by ethanol precipitation and washed with $70 \%$ ethanol. The air-dried DNA was dissolved in $100 \mu \mathrm{L}$ of Tris-EDTA (TE) buffer (10 mM Tris-HCl (pH 8.0) and $1 \mathrm{mM}$ EDTA). DNA concentrations were determined with a TapeStation 2200 system (Agilent, Santa Clara, CA, USA).

DNA amplification via the LAMP assay was performed according to the protocol described by Notomi et al. (2000, 2015) using a Loopamp DNA Amplification Kit (Eiken Chemical, Tokyo, Japan). Each reaction contained $12.5 \mu \mathrm{L}$ of reaction mix provided in the kit, $2 \mu \mathrm{L}$ of FIP3 primer $(20 \mu \mathrm{M}), 2 \mu \mathrm{L}$ of BIP3 primer $(20 \mu \mathrm{M}), 1 \mu \mathrm{L}$ of F3_3 primer $(5 \mu \mathrm{M}), 1 \mu \mathrm{L}$ of B3_3 primer $(5 \mu \mathrm{M}), 3.5 \mu \mathrm{L}$ of nuclease-free water, $1 \mu \mathrm{L}$ of Bst polymerase, and $2 \mu \mathrm{L}$ of DNA. The reactants were placed in a dedicated clean PCR tube, and reactions were performed at $60^{\circ} \mathrm{C}$ for $90 \mathrm{~min}$. Turbidity indicative of the reaction progress was recorded with an LA-320C turbidimeter (Eiken Chemical).

\section{Simplification of sample treatment for the LAMP assay}

To simplify the DNA extraction processes, we also evaluated DNA samples obtained by the following methods. When an excised leg was used, the sample was homogenized in $40 \mu \mathrm{L}$ of TE. Nucleases were inactivated by heating at $90{ }^{\circ} \mathrm{C}$ for $10 \mathrm{~min}$. Then, $2 \mu \mathrm{L}$ of the supernatant was used as a template for the LAMP reaction.

When a whole body was used, the sample was homogenized in $40 \mu \mathrm{L}$ of TE. It was allowed to settle for $1 \mathrm{~min}$, and $20 \mu \mathrm{L}$ of the supernatant was transferred to another tube and mixed with $200 \mu \mathrm{L}$ of InstaGene matrix (Bio-Rad, Hercules, CA, USA). The mixture was incubated at $56^{\circ} \mathrm{C}$ for $30 \mathrm{~min}$, vortexed for $10 \mathrm{~s}$, incubated at $100{ }^{\circ} \mathrm{C}$ for $8 \mathrm{~min}$, and then vortexed for $10 \mathrm{~s}$. After the insoluble material was precipitated for $5 \mathrm{~min}, 2 \mu \mathrm{L}$ of the supernatant was used as a LAMP reaction template.

\section{Analysis of mixed samples}

We also tested an extract from a mixed sample comprising one $S$. invicta worker and nine $F$. japonica workers. This native ant species is easily obtained and has a large amount of DNA per individual because of its large body size; so it is considered as an ideal sample for verifying the specificity of the LAMP method in the case of amplification using a contaminated DNA sample. In addition, about 10 ants can be homogenized in a microtube; so we chose to use one $S$. invicta and nine $F$. japonica workers in each microtube. The sample was homogenized in $400 \mu \mathrm{L}$ of TE, and the extract was allowed to settle for $1 \mathrm{~min}$ for precipitation. Then, $20 \mu \mathrm{L}$ of the supernatant was transferred to another tube and mixed with $200 \mu \mathrm{L}$ of InstaGene. The mixture was treated as described above, and $2 \mu \mathrm{L}$ of the supernatant was used as a template for the LAMP reaction.

\section{Results and discussion}

\section{Species specificity of the LAMP primers}

Among the seven primer sets designed, the FIP3 primer set showed the best amplification of $S$. invicta DNA. This primer set could amplify all DNA templates extracted from the $S$. invicta samples. The primer sequences are listed in Table 1, and their locations in the S. invicta COX1 gene are 
Table 1 The FIP3 primer set used for the LAMP reaction to detect Solenopsis invicta DNA

\begin{tabular}{lc}
\hline Primer & Sequence $\left(5^{\prime} \rightarrow 3^{\prime}\right)$ \\
\hline FIP3 & AGGCTCGTGTATCAACGTCAA \\
& GGTTTTATTGTTTGGGCACA \\
BIP3 & TGAATTTCCACTCTCCACGGA \\
& CCTATCGATCATCAGAGTGTG \\
F3_3 & TGCTATAATCGCTATCGGAT \\
B3_3 & CCTGTTAATCCTCCTATAGTGAAT \\
\hline
\end{tabular}

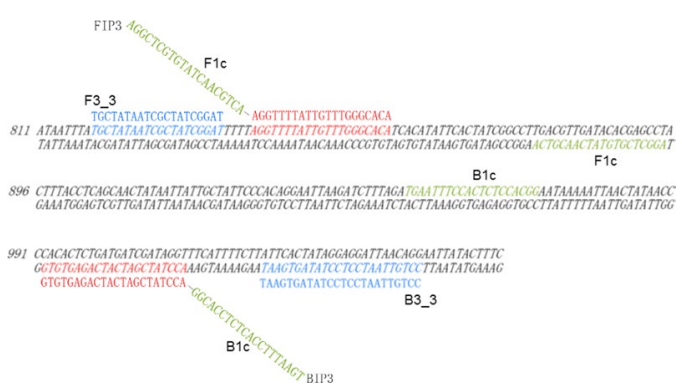

Fig. 1 Nucleotide sequences of the FIP3 primer set for the LAMP reaction and their locations in the $S$. invicta mitochondrial COXI gene. Italic indicates the nucleotide sequence of $S$. invicta mitochondrial COX1 DNA (NC_014672.1). The bases that correspond to COX1 DNA are colored as follows: red, $\mathrm{F} 2$ and $\mathrm{B} 2$ regions of FIP3 and BIP3; green: F1c and B1c regions of FIP3 and BIP3; and blue: F3_3 and B3_3

shown in Fig. 1. On the other hand, the other six primer sets were unable to detect some of the DNA templates.

We then performed LAMP assays with the FIP3 primer set for the eight Japanese native ant species (Fig. 2). Whereas marked turbidity was observed in the assay using $S$. invicta DNA, the diagnostic turbidity did not rise to the threshold level $(\mathrm{OD}=0.1)$ in the reactions with native species' DNA, indicating the specificity of the FIP3 set for S. invicta. Therefore, we selected the FIP3 set as the diagnostic primers.

\section{Utility of the LAMP assay for field samples}

To check for S. invicta at monitoring sites where their presence is assumed to be possible (e.g., port areas, green spaces, and playgrounds), we will occasionally need to use mixed samples of various ant species collected by traps. In some cases, we must identify $S$. invicta only from body parts, such as a leg. In addition, these DNA extracts should be obtained as easily as possible. Therefore, we tested the mixed DNA sample and single-leg DNA sample extracted by our rough protocol.

The assay using an extract from a mixed sample comprising one $S$. invicta worker and nine native ant workers clearly showed a positive turbidity signal for $S$. invicta (Fig. 3). Furthermore, our LAMP assay exhibited the same

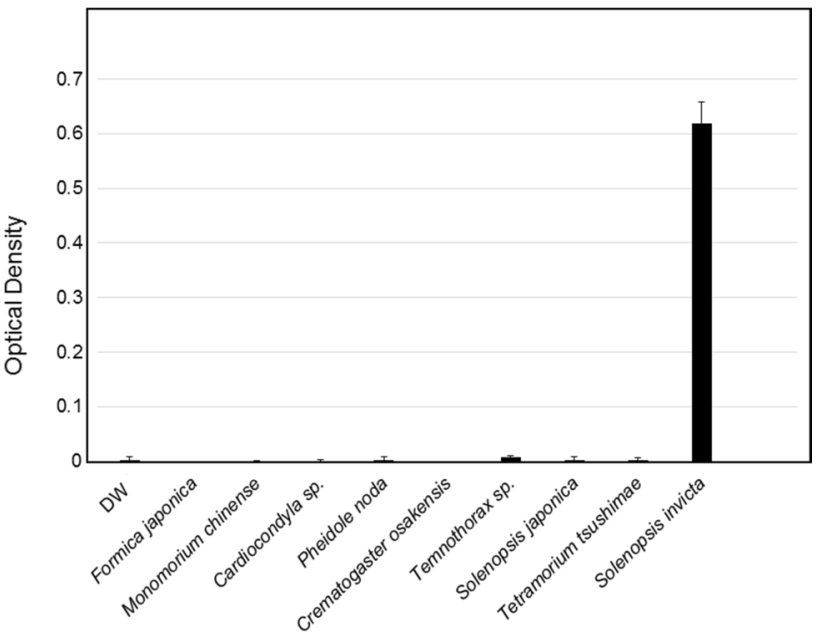

Fig. 2 Specificity of the LAMP reaction with the FIP3 primer set, as measured by the optical density of LAMP reactions containing RNase-free water as a negative control (DW), DNA of eight Japanese native ant species, and DNA of $S$. invicta. Error bars represent the standard deviation of three independent reactions

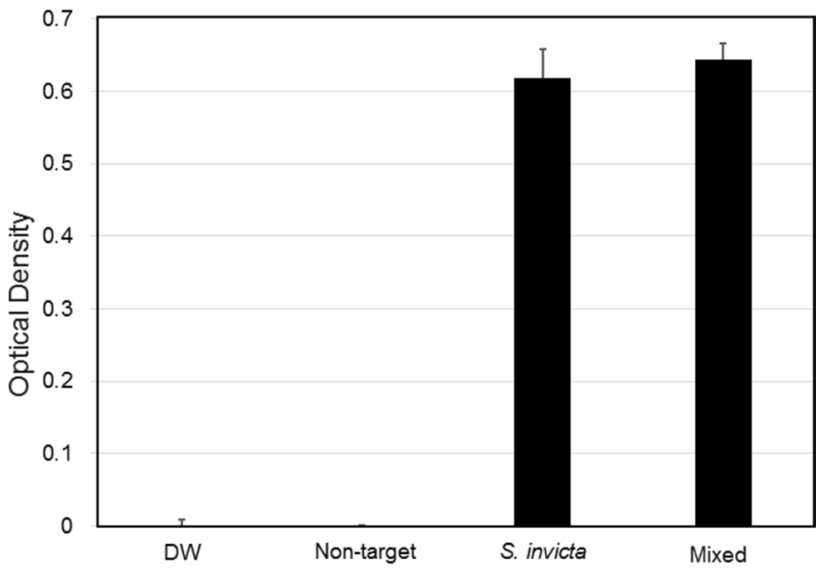

Fig. 3 Detection specificity of the LAMP reaction with the FIP3 primer set. The optical densities of LAMP reactions containing RNase-free water as a negative control (DW), whole-body extract of $F$. japonica (Non-target), whole-body extract of $S$. invicta ( $S$. invicta), or mixed extracts of one $S$. invicta worker and nine $F$. japonica workers (Mixed) are shown. Error bars represent the standard deviation of three independent reactions

positive signal for whole-body extract and leg extract of $S$. invicta (Fig. 4a). After completion of the LAMP reaction, the turbidity of the solution in the tube containing the DNA of $S$. invicta could be confirmed visually (Fig. 4b). These results indicate that our LAMP system with the FIP3 primer set does not need highly purified DNA and has sufficient specificity to detect one $S$. invicta individual out of 10 ants.

Of note, given the very high intraspecific variation within ant species distributed worldwide (Ascunce et al. 2011), our 


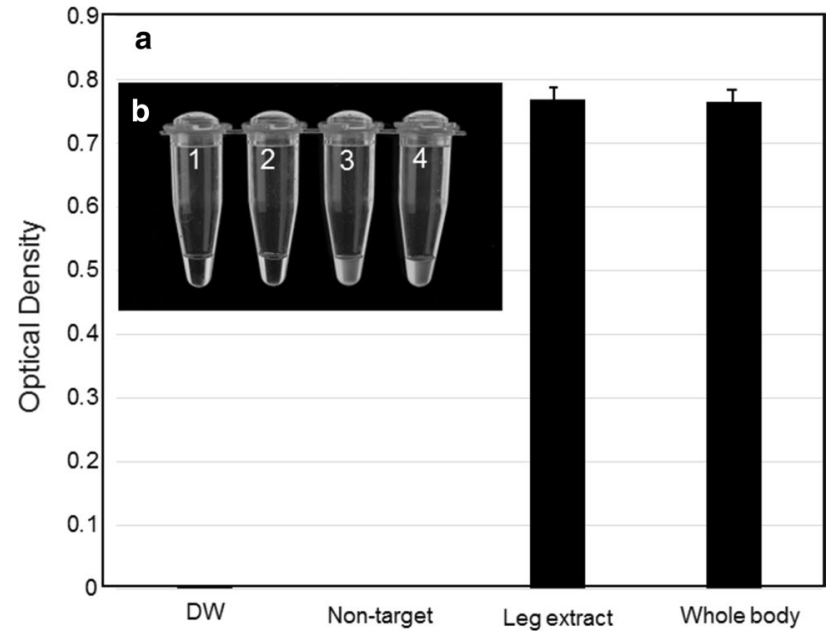

Fig. 4 Detection of S. invicta DNA in leg and whole-body extracts via the LAMP reaction with the FIP3 primer set. a Optical density of LAMP reactions containing RNase-free water as a negative control (DW), whole-body extract of Formica japonica (Non-target), leg extract of $S$. invicta (Leg extract), and whole-body extract of $S$. invicta (Whole body) are shown. Error bars represent the standard deviation of three independent reactions. b Turbidity after LAMP reactions in microtubes containing (1) RNase-free water as a negative control, (2) whole-body extract of $F$. japonica, (3) leg extract of $S$. invicta, and (4) whole-body extract of S. invicta

LAMP primers might be unable to amplify some $S$. invicta haplotypes that have never been detected in Japan. However, because all $S$. invicta individuals that have been collected so far were detectable, our LAMP primers are currently the most effective tool for the rapid detection of S. invicta in Japan. To improve the sensitivity and reliability of the LAMP assay system, it is necessary to monitor the DNA variation of S. invicta in Japan.

This method can be easily used by non-scientists. Thus, it can be used not only for quarantine in port areas but also for the detection of S. invicta in public spaces such as schoolyards and parks. We are preparing to distribute our S. invicta detection system to various organizations throughout Japan to construct a national fire ant monitoring network.
Acknowledgements We are grateful to Hitoshi Ohnishi of the National Institutes for Environmental Studies and the members of the Japan Wildlife Research Center for providing ant samples. We thank Mr. Seiji Narita for taking a beautiful picture of LAMP reactions. This research was supported by the Global Environment Research Fund (No. 4-1401, Leader: K. Goka) of the Ministry of the Environment, Japan.

Open Access This article is distributed under the terms of the Creative Commons Attribution 4.0 International License (http://creativeco mmons.org/licenses/by/4.0/), which permits unrestricted use, distribution, and reproduction in any medium, provided you give appropriate credit to the original author(s) and the source, provide a link to the Creative Commons license, and indicate if changes were made.

\section{References}

Ascunce MS, Yang CC, Oakey J, Calcaterra L, Wu WJ, Shih CJ, Goudet J, Ross KG, Shoemaker DW (2011) Global invasion history of the fire ant Solenopsis invicta. Science 331:1066-1068

Ide T, Kanzaki N, Masuya H, Okabe K (2018) Application of the LAMP assay for the detection of the Argentine ant, Linepithema humile (Hymenoptera: Formicidae), from captures of pan traps. Appl Entomol Zool 53:275-279

Nakajima N, Nakagawa N, Imaseki H (1988) Molecular size of woundinduced 1-aminocyclopropane-1-carboxylate synthase from Cucurbita maxima Duch. and change of translatable mRNA of the enzyme after wounding. Plant Cell Physiol 29:989-998

Notomi T, Okayama H, Masubuchi H, Yonekawa T, Watanabe K, Amino N, Hase T (2000) Loop-mediated isothermal amplification of DNA. Nucleic Acids Res 28:e63

Notomi T, Mori Y, Tomita N, Kanda H (2015) Loop-mediated isothermal amplification (LAMP): principle, features, and future prospects. J Microbiol 53:1-5

Valles SM, Porter SD (2003) Identification of polygyne and monogyne fire ant colonies (Solenopsis invicta) by multiplex PCR of Gp-9 alleles. Insect Soc 50:199-200

Valles SM, Strong CA, Callcott AMA (2016) Development of a lateral flow immunoassay for rapid field detection of the red imported fire ant, Solenopsis invicta (Hymenoptera: Formicidae). Anal Bioanal Chem 408:4693-4703

Publisher's Note Springer Nature remains neutral with regard to jurisdictional claims in published maps and institutional affiliations. 